\title{
Aplicativo para conferência e controle de cirurgia segura - princípios para segurança do paciente em centro cirúrgico
}

\author{
Application for conference and control of safe surgery - principles for patient safety in surgical \\ center
}

Solicitud de conferencia y control de cirugía segura - principios para la seguridad del paciente en el centro quirúrgico

Rafael Henrique Silva1*, Márcia Aparecida Nuevo Gatti², Jaqueline de Souza Lopes ${ }^{3}$, Rafael Gustavo Corbacho Marafon ${ }^{4}$, Eliane Bergo de Oliveira de Andrade ${ }^{1}$, Salazar Carmona de Andrade ${ }^{1}$.

\section{RESUMO}

Objetivo: Testar um aplicativo para conferência e controle de todas as etapas do checklist de cirurgia segura sugerido pela Organização Mundial da Saúde para a segurança do paciente submetido à cirurgia. Métodos: O trabalho utilizou de um aplicativo desenvolvido a segurança do paciente. Os resultados foram armazenados em banco de dados digital e analisados o tempo para aplicação do checklist, o nível de adesão, a porcentagem de etapas que foram realizadas e preenchidas corretamente e quais das etapas foram mais deficientes fazendo posteriormente uma comparação a literatura. Resultados: O aplicativo é uma ferramenta passível de ser utilizada, a adesão ao checklist de segurança do paciente precisa ser estimulada e a apresentação imediata do risco do paciente pode potencializar da cultura de cirurgia segura. Conclusão: $O$ aplicativo de cirurgia segura otimiza a utilização do checklist de cirurgia segura, alerta os profissionais sobre os riscos para a segurança do paciente, quantifica e apresenta em tempo real o risco para a segurança do paciente durante o procedimento cirúrgico.

Palavras-chave: Segurança do paciente, Procedimentos cirúrgicos operatórios, Smartphone.

\begin{abstract}
Objective: To test an application for checking and controlling all stages of the safe surgery checklist suggested by the World Health Organization for the safety of patients undergoing surgery. Methods: The study used an application developed for patient safety. The results were stored in a digital database and the time to apply the checklist, the level of adherence, the percentage of steps that were performed and completed correctly were analyzed and which of the steps were more deficient, making a comparison with the literature later. Results: The application is a tool that can be used, adherence to the patient safety checklist needs to be encouraged and the immediate presentation of the patient's risk can enhance the culture of safe surgery. Conclusion: The safe surgery application optimizes the use of the safe surgery checklist, alerts professionals about risks to patient safety, quantifies and presents in real time the risk to patient safety during the surgical procedure.
\end{abstract}

Key words: Patient safety, Operative surgical procedures, Smartphone.

\footnotetext{
${ }^{1}$ Hospital Universitário da Universidade Federal da Grande Dourados (HU-UFGD). Dourados - Mato Grosso do Sul. *E-mail: rafaelhenrique10@hotmail.com

${ }^{2}$ Centro Universitário Sagrado Coração. Bauru - São Paulo.

${ }^{3}$ Hospital Evangélico Dr. e Sra. Goldsby King. Dourados - Mato Grosso do Sul.

${ }^{4}$ Hospital Universitário da Universidade Federal do Mato Grosso do Sul (HU-UFMS). Campo Grande - Mato Grosso do Sul.
} 


\begin{abstract}
RESUMEN
Objetivo: Probar una aplicación para verificar y controlar todas las etapas de la Lista de verificación de cirugía segura sugerida por la Organización Mundial de la salud para la seguridad de los pacientes sometidos a cirugía. Métodos: El estudio utilizó una aplicación desarrollada para la seguridad del paciente. Los resultados se almacenaron en una base de datos digital y se analizó el tiempo para aplicar la lista de verificación, el nivel de adherencia, el porcentaje de pasos que se realizaron y completaron correctamente y cuáles de los pasos fueron más deficientes, haciendo una comparación con la literatura más adelante. Resultados: la aplicación es una herramienta que se puede utilizar, se debe alentar el cumplimiento de la lista de verificación de seguridad del paciente y la presentación inmediata del riesgo del paciente puede mejorar la cultura de la cirugía segura. Conclusión: la aplicación de cirugía segura optimiza el uso de la lista de verificación de cirugía segura, alerta a los profesionales sobre los riesgos para la seguridad del paciente, cuantifica y presenta en tiempo real el riesgo para la seguridad del paciente durante el procedimiento quirúrgico.
\end{abstract}

Palabras clave: Seguridad del paciente, Procedimientos quirúrgicos operativos, Smartphone.

\title{
INTRODUÇÃO
}

Estima-se que ocorra anualmente sete milhões de complicações cirúrgicas, destes, pelo menos dois milhões de pacientes morrem durante ou após o tratamento cirúrgico, sendo que evidências apontam que metade das complicações cirúrgicas são evitáveis (RIBEIRO HCTC, et al., 2017; AMAYA MR, et al., 2015). Outros estudos apontam que morre um a cada trezentos pacientes admitidos em hospitais e a causa da morte de mais de 50\% destes é relacionada a erros cirúrgicos evitáveis (MENDES W e MOURA ML, 2012; FERRAZ EM, 2009).

Em 2007 e 2008, a OMS escolheu a segurança dos cuidados cirúrgicos como a problemática a ser discutida e analisada com o desafio de conhecer quais são as principais complicações nos atos cirúrgicos. Esse trabalho resultou no Segundo Desafio Global para a Segurança do Paciente com o programa chamado Cirurgias Seguras Salvam Vidas com o objetivo geral de atenuar os eventos adversos decorrentes dos cuidados da assistência cirúrgica. E, no ano seguinte, a OMS elaborou e lançou um manual de orientações intitulado "Cirurgia Segura 2009: Cirurgias Seguras Salvam Vidas" (PAIVA ACR, et al., 2015; CORONA ARPD e PENICHE ACG, 2015; OMS, 2010).

Desta forma, a preocupação com a segurança do paciente cirúrgico ganha destaque mundial, com orientação para que seja instituída como uma política de saúde que deve ser implementada e monitorada com rigor em todos os serviços de saúde, tanto públicos quanto privados.Para o paciente que necessita realizar uma cirurgia é fundamental que os procedimentos anestésicos e cirúrgicos ocorram com qualidade e que a cultura de segurança do paciente possibilite que os possíveis erros sejam minimizados através da aplicação do Protocolo para Cirurgia Segura da OMS (CORONA ARPD e PENICHE ACG, 2015).

A proposta do Checklist de Cirurgia Segura é auxiliar as equipes cirúrgicas a seguirem, de forma sistemática, passos críticos de segurança, em todos os procedimentos cirúrgicos de qualquer hospital do mundo, independentemente do seu grau de complexidade, melhorando os padrões de segurança da assistência (PAIVA ACR, et al., 2015; FREITAS MR, et al., 2014).

O Checklist de Cirurgia Segura é essencial para a assistência ao paciente cirúrgico. Esse instrumento padroniza as ações realizadas pela equipe envolvida no procedimento, fortalece e estimula a cultura de segurança, além de funcionar como uma barreira para minimizar a ocorrência de riscos para os pacientes (OMS, 2010).

Segundo Arrais RF e Crotti PLR (2015), as tecnologias de informação e comunicação estão incorporadas em todos os campos de atuação humana, estimuladas principalmente pelo uso disseminado de smartphones com acesso à internet e favorecendo o desenvolvimento de aplicativos para os mais diversos ramos de atividades, seja para entretenimento ou suporte para atividades cotidianas, incluindo cuidados à saúde. 
A área da saúde certamente é uma das mais influenciadas pelo crescente uso das tecnologias de comunicação e informação. Com o passar dos anos, a utilização de dispositivos móveis cresceu e ganhou cada vez mais espaço nessa área, dando origem ao conceito de mobile health definido como práticas médicas e de saúde pública auxiliadas por aparatos portáteis (WORLD HEALTH ORGANIZATION, 2011).

Desta forma, o objetivo do trabalho é testar um aplicativo desenvolvido para conferência e controle de todas as etapas do checklist de cirurgia segura sugerido pela Organização Mundial da Saúde para a segurança do paciente submetido à cirurgia.

\section{MÉTODOS}

O trabalho utilizou um aplicativo desenvolvido para utilização em procedimentos cirúrgicos para potencializar a segurança do paciente. $O$ aplicativo foi baseado no Checklist de Cirurgia Segura estabelecido pela Organização Mundial da Saúde, com possibilidade de implantação em qualquer serviço de saúde e de fácil instalação em dispositivos móveis.

Para os testes, o aplicativo foi instalado em um tablet utilizado exclusivamente para a pesquisa. Essa prática justifica-se pela necessidade de evitar o uso de celulares nas salas de cirurgia. $O$ tablet sendo de uso restrito ao centro cirúrgico minimiza o risco de infecção.

O período de coleta ocorreu entre setembro e novembro de 2019 através da utilização do aplicativo desenvolvido. Participaram da pesquisa os profissionais médicos e de enfermagem responsáveis pelas cirurgias eletivas no Centro Cirúrgico que permitiram o teste do aplicativo durante o procedimento cirúrgico. Os profissionais foram previamente orientados sobre a utilização do aplicativo durante a cirurgia, participando apenas os que concordaram e assinaram o Termo de Consentimento Livre e Esclarecido - TCLE. A utilização do aplicativo não substituiu o preenchimento da lista de verificação impressa conforme rotina da instituição.

Após a realização dos testes, os resultados foram armazenados em banco de dados digital e analisados o tempo para aplicação do checklist, o nível de adesão ao checklist, à porcentagem de etapas que foram realizadas e preenchidas corretamente e a partir dos resultados apontados pelo próprio aplicativo, quais das etapas foram mais deficientes fazendo posteriormente uma comparação com as dificuldades na realização do checklist segundo a literatura.

O estudo foi realizado após aprovação do Comitê de Ética em Pesquisa em Seres Humanos da Universidade Sagrado Coração - Bauru/SP com parecer número 3.366.757.

\section{RESULTADOS}

O aplicativo produzido foi utilizado em 104 procedimentos de médio e grande porte de um Hospital Universitário. A tabela 1 apresenta a análise estatística do tempo, em segundos, utilizado para preencher a tela inicial do aplicativo com os dados de identificação do paciente.

Tabela 1 - Tempo em segundos desprendido para preencher a tela inicial do aplicativo.

\begin{tabular}{cccccc}
\hline Média & Mediana & Moda & $\begin{array}{c}\text { Desvio } \\
\text { Padrão }\end{array}$ & $\begin{array}{c}\text { Amplitude } \\
\text { Mínima }\end{array}$ & $\begin{array}{c}\text { Amplitude } \\
\text { Máxima }\end{array}$ \\
\hline 45 & 46 & 46 & 4 & 39 & 58 \\
\hline
\end{tabular}

Fonte: Silva $\mathrm{RH}$, et al., 2020.

Posteriormente foram analisadas cada uma das etapas do checklist de cirurgia segura presente no aplicativo, sendo elas Antes da Indução Anestésica, Antes da Incisão Cirúrgica e Antes do Paciente Sair da Sala. Em cada uma dessas etapas foram analisados a porcentagem dos itens preenchidos, o tempo gasto para o seu preenchimento e o risco para a segurança do paciente em cada uma dessas etapas, assim como levantado se houve significância estatística entre os resultados (Tabela 1). 
Tabela 2 - Avaliação dos itens questionados em todas as etapas do aplicativo.

\begin{tabular}{lcccc}
\hline \multicolumn{1}{c}{ Itens Avaliados } & \multicolumn{2}{c}{ Sim } & \multicolumn{2}{c}{ Não } \\
\cline { 2 - 5 } Antes da Indução Anestésica & $\mathbf{n .}$ & $\%$ & $\mathbf{n}$. & $\%$ \\
\hline Confirmado a identificação do paciente & 114 & 100,00 & - & - \\
Confirmado o local e procedimento cirúrgico & 114 & 100,00 & - & - \\
Termo de consentimento assinado & 95 & 83,33 & 19 & 16,66 \\
Sítio cirúrgico do lado correto/não se aplica & 96 & 84,21 & 18 & 15,78 \\
Checagem de equipamentos cirúrgicos & 114 & 100,00 & - & - \\
Oxímetro de pulso instalado e funcionando & 114 & 100,00 & - & - \\
Paciente tem alguma alergia & 18 & 15,78 & 96 & 84,21 \\
Risco de via aérea difícil e sem planejamento & - & - & 114 & 100,00 \\
Risco de perda sanguínea e sem planejamento & - & - & 114 & 100,00 \\
\hline Antes da Incisão Cirúrgica & & & & \\
\hline Todos os profissionais se identificaram & 23 & 22,11 & 81 & 77,88 \\
Cirurgião, anestesista e enfermagem confirmaram identificação, & 23 & 22,11 & 81 & 77,88 \\
local e procedimento & 23 & 22,11 & 81 & 77,88 \\
Revisão realizada pelo cirurgião & 23 & 22,11 & 81 & 77,88 \\
Revisão realizada pelo anestesista & 23 & 22,11 & 81 & 77,88 \\
Revisão realizada pela enfermagem & 34 & 32,69 & 70 & 67,30 \\
Antibiótico profilático adequado & 104 & 100,00 & - & - \\
Exames de imagem disponível & & & & \\
\hline Antes do Paciente Sair da Sala de Cirurgia & 104 & 100,00 & - & - \\
\hline Confirmado o procedimento realizado & 104 & 100,00 & - & - \\
Contagem de compressas e instrumentais e agulhas & 104 & 100,00 & - & - \\
Biópsias identificadas & - & - & 104 & 100,00 \\
Problemas com equipamentos & - & - & 104 & 100,00 \\
Cirurgião, anestesista e enfermagem analisaram principais & & &
\end{tabular}

Fonte: Silva RH, et al., 2020.

Em todos os procedimentos em que o aplicativo foi testado, a etapa Antes de Sair da Sala de Cirurgia foi realizada conforme preconizado, o paciente ainda na sala cirúrgica. Nota-se que as respostas foram as mesmas em todos os casos, a maioria dos itens foram realizados, no entanto, em nenhum momento houve uma reunião entre enfermagem, anestesista e cirurgião para discutir sobre os principais pontos do pósoperatório dos pacientes. Antes da Indução Anestésica seis itens eram passíveis de serem respondidos adequadamente (Tabela 3).

Tabela 3 - Tempo desprendido, em segundos, para realizar a etapa Antes da Indução Anestésica.

\begin{tabular}{lcccccccc}
\hline & n. & Média & Mediana & Moda & $\begin{array}{c}\text { Desvio } \\
\text { Padrão }\end{array}$ & $\begin{array}{c}\text { Amplitude } \\
\text { Mínima }\end{array}$ & $\begin{array}{c}\text { Amplitude } \\
\text { Máxima }\end{array}$ & $\begin{array}{c}\mathbf{P} \\
\text { Valor }\end{array}$ \\
\hline $\begin{array}{l}\text { Todos os } \\
\text { procedimentos }\end{array}$ & 114 & 68 & 60 & 56 & 18 & 46 & 112 & $<0,001$ \\
$\begin{array}{l}\text { Os 6 itens } \\
\text { respondidos }\end{array}$ & 59 & 55 & 56 & 56 & 5 & 46 & 64 & $<0,001$ \\
$\begin{array}{l}\text { Os 6 itens } \\
\text { respondidos } \\
\text { paciente tem }\end{array}$ & 18 & 77 & 77 & 75 & 2 & 70 & 80 & $<0,001$ \\
$\begin{array}{l}\text { alergia } \\
\begin{array}{l}\text { Apenas 5 itens } \\
\text { respondidos }\end{array}\end{array}$ & 17 & 81 & 81 & 84 & 3 & 75 & 85 & $<0,001$ \\
$\begin{array}{l}\text { Apenas 4 itens } \\
\text { respondidos }\end{array}$ & 10 & 108 & 108 & 106 & 3 & 104 & 112 & $<0,001$ \\
\hline
\end{tabular}

Legenda: $\mathrm{P}$ valor $<0,001$ indica que há significância estatística entre os grupos

Fonte: Silva $\mathrm{RH}$, et al., 2020. 
Nos casos em que o aplicativo foi utilizado e o paciente referia alergia, optou-se por distingui-lo dos casos em que não havia essa especificidade, pois, listar no aplicativo as substâncias alérgicas demandavam de um maior tempo.

Os resultados mostram que quanto maior o número de itens respondidos, menor o tempo gasto para preencher o checklist, mostrando que a partir do momento que se tem conhecimento das informações e os dados estão disponíveis, o uso do aplicativo otimiza o serviço.

A Tabela 4 apresenta a análise estatística do tempo gasto, em segundos, para realizar a etapa Antes da Incisão Cirúrgica. Nessa etapa são sete itens que devem ser realizados conforme o aplicativo de cirurgia segura desenvolvido.

Tabela 4 - Tempo desprendido, em segundos, para realizar a etapa Antes da Incisão Cirúrgica.

\begin{tabular}{|c|c|c|c|c|c|c|c|c|}
\hline & n. & Média & Mediana & Moda & $\begin{array}{l}\text { Desvio } \\
\text { Padrão }\end{array}$ & $\begin{array}{l}\text { Amplitude } \\
\text { Mínima }\end{array}$ & $\begin{array}{c}\text { Amplitude } \\
\text { Máxima }\end{array}$ & $\begin{array}{c}\mathbf{P} \\
\text { Valor }\end{array}$ \\
\hline $\begin{array}{l}\text { Todos os } \\
\text { Procedimentos }\end{array}$ & 114 & 41 & 38 & 37 & 7 & 35 & 66 & $<0,001$ \\
\hline Os 7 itens respondidos & 23 & 53 & 53 & 49 & 5 & 41 & 66 & $<0,001$ \\
\hline $\begin{array}{l}\text { Apenas } 2 \text { itens } \\
\text { respondidos }\end{array}$ & 11 & 37 & 37 & 36 & 1 & 36 & 39 & $<0,001$ \\
\hline $\begin{array}{l}\text { Apenas } 1 \text { item } \\
\text { respondido }\end{array}$ & 70 & 37 & 37 & 37 & 2 & 35 & 40 & $>0,05$ \\
\hline
\end{tabular}

Legenda: $\mathrm{P}$ valor $<0,001$ indica que há significância estatística entre os grupos

Fonte: Silva $\mathrm{RH}$, et al., 2020.

A última etapa do aplicativo, conforme o checklist da OMS é antes do paciente sair da sala de cirurgia. Os resultados foram os mesmos em todos os casos, o único item que não foi realizado adequadamente foi o que se refere ao cirurgião, anestesista e enfermagem analisaram principais pontos do pós-operatório.

A tabela a seguir apresenta o risco para a segurança do paciente em cada uma das etapas do checklist, antes da indução anestésica, antes da incisão cirúrgica e antes do paciente sair da sala e assim associar a quantidade de itens não realizados e a risco percentual para o paciente calculado pelo aplicativo.

Tabela 5 - Aumento percentual para a segurança do paciente pela não realização adequada do checklist de cirurgia segura.

\begin{tabular}{lcc}
\hline \multicolumn{1}{c}{ Etapas do Checklist } & $\begin{array}{c}\text { Percentual do } \\
\text { checklist realizado }\end{array}$ & $\begin{array}{c}\text { Aumento do risco percentual } \\
\text { para a segurança do paciente }\end{array}$ \\
\hline Antes da Indução Anestésica & & \\
Todos os 6 itens preenchidos & $100,00 \%$ & $00 \%$ \\
\hline 1 item não preenchido & $83,33 \%$ & $06 \%$ \\
2 itens não preenchidos & $66,66 \%$ & $11 \%$ \\
\hline Antes da Incisão Cirúrgica & & $00 \%$ \\
\hline Todos os 7 itens preenchidos & $100,00 \%$ & $24 \%$ \\
5 itens não preenchidos & $28,57 \%$ & $29 \%$ \\
\hline Itens não preenchidos & $14,28 \%$ & $00 \%$ \\
\hline Antes do Paciente Sair da Sala & & $20 \%$ \\
\hline Todos os 5 itens preenchidos & $100,00 \%$ & \\
\hline 1 item não preenchido & $80,00 \%$ & \\
\hline
\end{tabular}

Fonte: Silva $\mathrm{RH}$, et al., 2020.

\section{DISCUSSÃO}

Os resultados apresentados pelo aplicativo evidenciaram que a realização de todos as etapas do checklist de cirurgia segura é um desafio para os profissionais e instituições de saúde. O aplicativo é uma ferramenta que pode permitir novos horizontes para a cirurgia segura. 
O checklist além de uma ferramenta para garantir segurança ao paciente, também é uma forma importante de melhorar a comunicação. Alguns profissionais têm dificuldade de verbalizar informações, no entanto, o checklist oportuniza que os profissionais compartilhem eventuais preocupações com os demais membros da equipe (PANCIERI AP, et al., 2014; CUNAT C, et al., 2011; HAYNES AB, et al., 2011; PAUGAM-BURTZ C e GUERRERO O, 2011; RATEAU $F$, et al., 2011). O recomendado é que um único profissional seja o responsável por liderar a aplicação do checklist que precisa ser de preenchimento rápido e fácil. Em relação ao o tempo utilizado para sua aplicação, ele deve ser de aproximadamente três minutos (SOUZA RM, et al., 2016; PANCIERI AP, et al., 2014).

A identificação correta do paciente é válida e necessária para que toda a equipe cirúrgica não execute procedimentos errôneos (ALPENDRE FT, et al., 2017; FREITAS MR, et al., 2014). O aplicativo verificou que essa identificação ocorreu em $100 \%$ dos procedimentos. Identificar o paciente é fundamental para garantir que a assistência seja prestada ao paciente correto, considerando a possibilidade de troca de paciente. Diante desta possibilidade, a identificação do paciente, segundo protocolo, é preconizado pelo Ministério de Saúde para todas as instituições que prestam assistência à saúde (AMAYA MR, et al., 2015; MINISTÉRIO DA SAÚDE, 2013).

A confirmação do local e do procedimento foi realizada em todos os procedimentos em que o aplicativo foi utilizado. A confirmação do procedimento, local e lateralidade da cirurgia, assim como do paciente correto, quando realizada são itens passíveis de intervenção e garantem maior segurança ao paciente (PAIVA ACR, et al., 2015). Garcia TF e Oliveira AC (2018) evidenciam que a confirmação do local e procedimento cirúrgico, acrescido da identificação correta do paciente são medidas reconhecidas e que reduzem em cerca de $50 \%$ as complicações evitáveis.

A autorização para cirurgia ou termo de consentimento cirúrgico é uma exigência assegurada por lei onde constam informações detalhadas sobre o procedimento e os riscos inerentes à cirurgia. É um documento necessário para cirurgias eletivas e confere uma proteção tanto para o paciente quanto para 0 cirurgião (STUDART RMB, et al., 2017).

O aplicativo questiona o profissional se o termo de consentimento está assinado, em $83,33 \%$ dos casos em que 0 aplicativo foi testado a resposta foi afirmativa. Entende-se que a ausência do termo de consentimento assinado deve ser um impedimento para o procedimento.

A fim de se evitar falhas no processo, o checklist de cirurgia segura conta com uma etapa questionando se o sítio cirúrgico está devidamente demarcado. Essa demarcação é feita antes da indução anestésica e através da marcação do local cirúrgico e da lateralidade com um marcador de tinta permanente (PAIVA ACR, et al., 2015).

O aplicativo de cirurgia segura possibilitou que identificar que em $84,21 \%$ dos procedimentos o sítio cirúrgico estava demarcado. A demarcação é uma segurança para o paciente e para toda a equipe envolvida no procedimento. Mesmo o paciente confirmando o local do procedimento, a demarcação do sítio cirúrgico é importante, visto que minimiza o risco de uma falha durante o processo.

Um dos pontos do checklist da cirurgia segura é a verificação da disponibilidade e funcionalidade dos equipamentos que serão utilizados na realização do procedimento. Essa ação deve ser realizada antes da cirurgia e caso seja constatado algum problema, o enfermeiro responsável pelo Centro Cirúrgico precisa ser comunicado e as devidas providências tomadas para garantir a segurança do paciente (STUDART RMB, et al., 2017; PANCIERI AP, et al., 2014).

A checagem se o oxímetro de pulso estava corretamente instalado no paciente e se a leitura era apresentada no monitor foi observado em $100 \%$ dos procedimentos onde o aplicativo foi utilizado. A verificação do oxímetro de pulso é uma preocupação das equipes cirúrgicas. Entre os equipamentos de monitorização, o oxímetro é particularmente importante por permitir a identificação precoce da ocorrência de eventos respiratórios, sendo este, um dos principais incidentes relacionados à mortalidade em anestesia (ALMEIDA RE e RODRIGUES MCS, 2018; KIM RY, et al., 2015; FREITAS MR, et al., 2014). 
Identificar se o paciente possui algum tipo de alergia é importante para garantir a segurança. Essa prática permite evitar que ocorram manifestações clínicas e qualquer comprometimento durante o procedimento cirúrgico. O registro dessa informação é necessário e deverá contemplar qualquer sensibilidade a medicamentos, alimentos ou materiais e ainda, as reações alérgicas prévias (PAIVA ACR, et al., 2015).

A avaliação pré-anestésica oportuniza a identificação prévia de condições clínicas desfavoráveis para a cirurgia. Nesse contexto, ações de prevenção de danos estão associadas à segurança na administração de medicamentos, incluindo os anestésicos. A adoção de uma avaliação pré-anestésica sistemática resulta em uma baixa incidência de complicações anestésicas. Deve-se destacar que uma cirurgia nunca deve ser realizada caso haja alguma inconformidade (AMAYA MR, et al., 2015; SCHWARTZMAN UP, et al., 2011)

$O$ protocolo de cirurgia segura orienta que a equipe cirúrgica discuta previamente sobre os riscos de eventos críticos durante o procedimento. A transfusão sanguínea é importante e tem como objetivo manter o volume sanguíneo, os níveis de hemoglobina e fatores de coagulação propícios para um procedimento cirúrgico seguro. A reserva de hemocomponentes no pré-operatório deve ocorrer quando há risco de perda sanguínea superior a $500 \mathrm{ml}$ em adultos ou $7 \mathrm{ml}$ por quilo de peso em crianças. (AMAYA et al, 2015; OMS, 2010).

A OMS reforça a importância desse item no checklist e recomenda que um membro da equipe seja o responsável por confirmar a disponibilidade de hemoderivados para a cirurgia, sempre que necessário. (PAIVA et al, 2015; OMS, 2010).

O protocolo de cirurgia segura orienta que a equipe cirúrgica discuta previamente sobre os riscos de eventos críticos durante o procedimento. A recomendação é que, sempre que necessário, o planejamento cirúrgico seja revisado e considerado a previsão de reposição de fluidos e reservas de hemocomponentes (AMAYA MR, et al., 2015).

Antes que ocorra a incisão cirúrgica há um momento denominado time out, ou pausa cirúrgica. Nesse momento, todos os profissionais devem interromper suas atividades e confirmar a identificação do paciente, o local e o procedimento cirúrgico que será realizado (MAZIERO ECS, et al., 2015). O aplicativo desenvolvido questiona se o time out foi realizado. Caso não tenha ocorrido, o aplicativo adverte com uma mensagem que o esse fato é um risco para a segurança do paciente.

O time out é uma das ações mais importantes do checklist de cirurgia segura. Nesse momento, todos os profissionais devem realizar a confirmação de dados importantes como a identificação do paciente a ser operado, a cirurgia que será realizada e qual o lado a ser operado. No entanto, essa prática ainda é incomum de acontecer durante os procedimentos (GARCIA TF e OLIVEIRA AC, 2018; THE JOINT COMMISION, 2017; MOLINA G, et al., 2016).

A profilaxia antimicrobiana deve ser administrada no período de 60 minutos antes da incisão cirúrgica para que o risco de infecção do sítio cirúrgico seja minimizado. Esse tempo precisa ser respeitado para que se alcance o maior nível terapêutico do antibiótico no momento de maior exposição tecidual aos microrganismos (AMAYA MR, et al., 2015; ANVISA, 2013).

Os exames de imagem estavam disponíveis em $100 \%$ dos procedimentos em que o aplicativo foi utilizado, eles são fundamentais para que seja alcançado uma boa evolução do paciente no trans e pós-operatório. Os exames podem alertar os profissionais de saúde para a necessidade de monitorizar condições clínicas específicas que possam acarretar alterações durante o procedimento cirúrgico (PAIVA ACR, et al., 2015).

Antes do paciente sair da sala cirúrgica é importante conferir todos os documentos referentes à cirurgia, além de registrar as recomendações especiais para recuperação pós-operatória (MAZIERO ECS, et al., 2015). O aplicativo questiona os profissionais sobre todas as conferências preconizadas pela OMS e alerta se alguma orientação está sendo infringida.

Como forma de garantir a segurança do paciente, a OMS preconiza que o processo de contagem de compressas, instrumentais e perfurocortantes deve ser realizado em qualquer cirurgia, na qual esses 
materiais possam ficar retidos em cavidades dos pacientes. A verificação deve ser realizada pela equipe cirúrgica e a responsabilidade é de todos os profissionais envolvidos no procedimento (FREITAS PS, et al., 2016).

O checklist de cirurgia segura aponta a obrigatoriedade de realizar a identificação de qualquer peça anatômica obtida durante a cirurgia. É fundamental a identificação correta das biópsias, sem que haja necessidade de novo procedimento ou gerar dúvidas quanto aos direcionamentos para sequência do tratamento, sendo a falta de comunicação entre a equipe uma razão para essa ocorrência (BOHOMOL E e TATARLI JA, 2017).

O aplicativo desenvolvido além de ser uma ferramenta para nortear a checagem pela equipe quantifica esse risco. A partir do momento que o profissional visualiza que um único item não realizado aumenta o risco do paciente em $7 \%$ espera-se que ele possa rever a sua conduta. Quantificar o aumento do risco é uma ferramenta que pode ser utilizada em ações de atualização e treinamento para a conscientização dos profissionais.

Nas situações que há uma baixa adesão à maioria dos itens do protocolo, constata-se a inconsistência das informações e baixa qualidade no processo de segurança do paciente cirúrgico. A educação permanente é fundamental para disseminar práticas de segurança, estabelecer uma cultura de segurança envolve gestão, lideranças, trabalho em equipe, comunicação efetiva, crenças individuais, além de cultura não punitiva e de aprendizado com os erros (ALMEIDA RE e RODRIGUES MCS, 2018; TAVARES APM, et al., 2018; CARMO TG, et al., 2017).

Ferramentas eletrônicas podem ser um facilitador para os profissionais de saúde, porém, essas ferramentas só irão ajudar a melhorar da qualidade da assistência se os profissionais forem sensibilizados quanto à importância do preenchimento completo do instrumento, com participação efetiva e respostas conscientes de toda a equipe envolvida. O checklist deve ser uma estratégia de segurança e não apenas mais uma lista obrigatória de verificação de itens antes, durante e após as cirurgias (RIBEIRO HCTC, et al., 2017).

\section{CONCLUSÃO}

As tecnologias aplicadas a área da saúde estão se tornando cada vez mais presente no cotidiano dos profissionais de saúde. $O$ aplicativo de cirurgia segura otimiza a utilização do checklist de cirurgia segura, alerta os profissionais sobre os riscos para a segurança do paciente, quantifica e apresenta em tempo real o risco para a segurança do paciente durante o procedimento cirúrgico. O aplicativo é uma ferramenta testada, viável e passível de ser utilizada por outros profissionais e instituições interessados.

\section{REFERÊNCIAS}

1. ALMEIDA RE, RODRIGUES MCS. Preenchimento da Lista de Verificação de Segurança Cirúrgica em hospitais brasileiros. Rev Rene. 2018.

2. ALPENDRE FT, et al. Ciclo PDCA para elaboração de checklist de segurança cirúrgica. Cogitare Enfermagem 2017 (22)3: e50964.

3. AMAYA MR, et al. Análise do registro e conteúdo de checklists para cirurgia segura. Esc Anna Nery 2015; 19(2):246251.

4. ANVISA. Agência Nacional de Vigilância Sanitária. Resolução RDC no 36, de 25 de Julho de 2013. Institui ações para segurança do paciente em serviços de saúde e dá outras providências. Diário Oficial da União 2013.

5. ARRAIS RF, CROTTI PLR. Revisão: aplicativos para dispositivos móveis ("Apps") na automonitorização em pacientes diabéticos. J. Health Inform. 2015; 7(4):127-33

6. BOHOMOL E, TATARLI JA. Utilização de cenários para a educação sobre segurança do paciente em centro cirúrgico. Rev. SOBECC, São Paulo. jul./set. 2017; 22(3): 138-144.

7. CARMO TG, et al. The implementation process of the safe surgery protocolo. Rev Cubana Enfermagem 2017. 
8. CORONA ARPD, PENICHE ACG. A Cultura de Segurança do Paciente na Adesão ao Protocolo de Cirurgia Segura. Rev. SOBECC, São Paulo. Jul/set. 2015; 20(3): 179-185

9. CUNAT C, et al. Stratégie de deployment de la checklist dans un CHU. Ann Fr Anesth Reanim. 2011; 30(6):484-8.

10. FERRAZ EM. A cirurgia segura. Uma exigência do século XXI. Rev Col Bras Cir. 2009; 36:281-2.

11. FREITAS MR, et al. Avaliação da adesão ao checklist de cirurgia segura da OMS em cirurgias urológicas e ginecológicas, em dois hospitais de ensino de Natal, Rio Grande do Norte, Brasil. Cad. Saúde Pública. 2014.

12. FREITAS PS, et al. Processo de contagem cirúrgica: evidências para a segurança do paciente. Rev Gaúcha Enferm. 2016.

13. GARCIA TF, OLIVEIRA AC. Índice autorreferido pela equipe de cirurgia ortopédica sobre o protocolo e checklist de cirurgia segura. Cogitare Enferm. 2018; (23)1: e52013.

14. HAYNES $A B$, et al. Changes in safety attitude and relationship to decreased postoperative morbidity and mortality following implementation of a checklist-based surgical safety intervention. BMJ Qual Saf. 2011; 20:102-7.

15. KIM RY, et al. Sustainability and longterm effectiveness of the WHO Surgical Safety Checklist combined with pulse oximetry in a resource-limited setting: two-year update from Moldova. JAMA Surg. 2015; 150(5):473-9.

16. MAZIERO ECS, et al. Adesão ao uso de um checklist cirúrgico para segurança do paciente. Rev Gaúcha Enferm. 2015 dez;36(4):14-20.

17. MENDES W, MOURA MLO. Avaliação de eventos adversos cirúrgicos em hospitais do Rio de Janeiro. Rev Bras Epidemiol 2012; 15:523-35.

18. MINISTÉRIO DA SAÚDE. Agência Nacional de Vigilância Sanitária. Portaria no 2095, de 24 de setembro de 2013. Aprova os protocolos básicos de segurança do paciente. Brasília: Ministério da Saúde; 2013.

19. MOLINA G, et al. Implementation of the Surgical Safety Checklist in South Carolina Hospitals is associated with improvement in perceived perioperative safety. J Am Coll Surg. 2016;222(5).

20. OMS (Organização Mundial da Saúde). Segundo desafio global para segurança do paciente: cirurgia seguras salvam vidas. Rio de Janeiro: Organização Pan-Americana da Saúde (OPAS) e Ministério da Saúde (MS); Agência Nacional de Vigilância Sanitária (ANVISA); 2010.

21. PAIVA ACR, et al. Checklist de cirurgia segura: análise do preenchimento da ficha de verificação no pré, trans e pósoperatório. Rev. Enfermagem 2015; v. 18 n.02.

22. PANCIERI AP, et al. Aplicação do checklist para cirurgia segura: Relato de experiência. Rev. SOBECC, São Paulo. jan./mar. 2014; 19(1): 26-33.

23. PAUGAM-BURTZ C, GUERRERO O. Check-list sécurité au bloc opératoire: le bilan après un an de deployment à I'hôpital Beaujon. Ann Fr Anesth Reanim. 2011; 30(6):475-8.

24. RATEAU F, et al. Check-list "Patient Safety" in the operating room: one year experience of 40,000 surgical procedures at the university hospital of Nice. Ann Fr Anesth Reanim 2011; 30:479-83.

25. RIBEIRO HCTC, et al. Adesão ao preenchimento do checklist de segurança cirúrgica. Cad. Saúde Pública 2017; 33(10).

26. SCHWARTZMAN UP, et al. Complicações anestésicas em cirurgia plástica e a importância da consulta préanestésica como instrumento de segurança. Rev. Bras. Cir. Plast. 2011.

27. SOUZA RM, et al. Aplicabilidade do checklist de cirurgia segura em centros cirúrgicos hospitalares. Rev. Sobecc, São paulo. Out./dez. 2016; 21(4): 192-197.

28. STUDART RMB, et al. Avaliação sobre a segurança do paciente durante o procedimento anestésico-cirúrgico. Rev enferm UFPE online. Recife: 2017; 2195-201.

29. TAVARES APM, et al. Patient safety culture from the perspective of the nursing team. Rev Rene. 2018; 19:e3152.

30. THE JOINT COMMISSION. National Patient Safety Goals Effective January 2017. Office-Based Surgery Accreditation Program. TJC. 2017.

31. WORLD HEALTH ORGANIZATION. Global Observatory for eHealth. mHealth, New Horizons for Health trough Mobile Technologies. Geneva (Switzerland): WHO; 2011. 